\title{
Ionoluminescence in the helium ion microscope
}

Stuart A. Boden*‡, Thomas M. W. Franklin†, Larry Scipioni\#, Darren M. Bagnall* and Harvey N. Rutt*

* Electronics and Computer Science, University of Southampton, Highfield, Southampton, SO17 1BJ, UK

tOptoelectronics Research Centre, University of Southampton, Highfield, Southampton, SO17 1BJ, UK

\#Carl Zeiss SMT, Inc., One Corporation Way, Peabody, Massachusetts, 01960, USA

łcorresponding author, tel: +44 (0)23 8059 3737, fax: +44 (0)23 8059 3029, email:

sb1@ecs.soton.ac.uk

Key words: Cathodoluminescence, ionoluminescence, helium ion microscopy (HIM), scanning electron microscopy (SEM), yttrium aluminium garnet (YAG), rare earth, quantum dots 


\begin{abstract}
lonoluminescence (IL) is the emission of light from a material due to excitation by an ion beam. In this work, a helium ion microscope (HIM) has been used in conjunction with a luminescence detection system to characterize IL from materials in an analogous way to how cathodoluminescence $(C L)$ is characterized in a scanning electron microscope (SEM). A survey of the helium ion beam induced IL characteristics, including images and spectra, of a variety of materials known to exhibit $\mathrm{CL}$ in an SEM is presented. Direct bandgap semiconductors that luminesce strongly in the SEM, are found not do so in the HIM, possibly due to defect-related non-radiative pathways created by the ion beam. Other materials do, however, exhibit IL, including a cerium-doped garnet sample, quantum dots and rare earth doped $\mathrm{LaPO}_{4}$ nanocrystals. These emissions are a result of transitions between $\mathrm{f}$ electron states or transitions across size dependent band gaps. In all these samples, IL is found to decay with exposure to the beam, fitting well to double exponential functions. In an exploration of the potential of this technique for biological tagging applications, imaging with the IL emitted by rare earth doped $\mathrm{LaPO}_{4}$ nanocrystals, simultaneously with secondary electron imaging, is demonstrated at a range of magnifications.
\end{abstract}

\section{Introduction}

Helium ion microscopy (HIM) is a new surface imaging technique, similar to scanning electron microscopy (SEM) but based on a focused beam of helium ions rather than electrons (Scipioni et al., 2008; Ward et al., 2006). Helium atoms are ionised at the tip of an atomically-sharp tungsten needle (the source) and accelerated down the column of the microscope, towards a sample through an aperture and a set of lenses which focus the beam producing a sub-nm spot size at the sample surface. The beam is scanned across the sample, generating secondary electrons that are detected by an in-chamber Everhart-Thornley (ET) detector, constructing an image of the sample surface, pixel by pixel. The larger mass and therefore shorter De Broglie wavelength of helium ions compared to electrons, together with the bright and atomically sharp source, allows the beam of helium ions to be focused to a smaller spot on the sample compared to a beam of electrons in an SEM. As the helium beam enters a sample, the ions lose energy primarily through inelastic scattering with the sample electrons, leading to a high SE yield without significantly changing the propagation direction of the incident ions (Cohen-Tanugi \& Yao, 2008). This ensures that the primary beam remains tightly focused as it passes through the first few tens of nanometers of the sample, leading to a small interaction volume and therefore a high resolution. A probe size of $0.25 \mathrm{~nm}$ has been demonstrated by the microscope manufacturers (Scipioni, 2008; Vanden Berg-Foels et al., 2012). The latest HIM systems, including the one used for this work, are specified to an edge resolution, measured across the edge of a graphene flake, of $<0.35 \mathrm{~nm}$.

In addition to generating secondary electrons, the He ion beam can also cause the emission of light due to the relaxation of excited sample electrons. This process is known as ionoluminescence (IL) and is analogous to the phenomenon of cathodoluminescence (CL) in SEM, the emission of light resulting from the excitation of sample electrons by an electron beam. The first applications of $\mathrm{CL}$ were in mineralogy and petrology where the $\mathrm{CL}$ emission gives an indication of rare earth and transition metal content (Smith \& Stenstrom, 1965). CL is also widely used in the study of semiconductors, due to the strong emission of light possible as a result 
of radiative recombination of electron-hole pairs generated by the primary beam (Yacobi \& Holt, 1986). The emission can be intrinsic in nature, i.e. as a result of a direct recombination across the band gap of the material, or extrinsic, as a result of recombination via impurity or defect states. This technique has found applications in imaging of defects through contrast provided by increased non-radiative recombination at the defect location or emission of light from dislocations (Pennycook, 2008). Determination of minority carrier lifetimes and surface recombination velocities is also possible with time-resolved $\mathrm{CL}$ (Hastenrath \& Kubalek, 1982). In addition, $\mathrm{CL}$ at low temperatures can be used to probe bound electron-hole pairs or excitons in a semiconductor (Wakefield et al., 1984). By generating luminescence in a HIM rather than an SEM, it is hoped that the higher resolution capabilities can be exploited to yield a higher level of detail in the study of defects in semiconductors. More recently, $\mathrm{CL}$ has been employed for biological applications in a similar way to confocal microscopy of fluorescentlytagged samples (Fisher et al., 2008; Fisher et al., 2010). Luminescent species including dyes and quantum dots are used to label antibodies which attach to specific proteins on biological cells. Collecting $\mathrm{SE}$ and $\mathrm{CL}$ images simultaneously enables the mapping of the location of the labelled molecules on the cell. As the move from confocal microscopy to SEM led to a resolution increase and so an enhancement in the ability to specify the location of labelled molecules on a cell, it is hoped that applying similar techniques in HIM will lead to a further enhancement due to the higher resolution of the instrument.

Imaging using ion-induced photon emission in a HIM was briefly mentioned in a paper by Scipioni et al. where an example of a low magnification (field of view (FOV) $=1350 \mu \mathrm{m}$ ) IL image of salt crystals was presented (Scipioni et al., 2007). This work represents a more detailed survey of ionoluminescence imaging and spectroscopy using a HIM combined with a state-of-the-art luminescence detection system. Thin film and bulk samples of direct band gap semiconductors, a rare earth doped ceramic, quantum dots and rare-earth doped nanocrystals are studied, all of which are known to give strong $\mathrm{CL}$ emission in SEM.

\section{Experimental Method}

A helium ion microscope (HIM, Carl Zeiss Orion Plus) equipped with a luminescence detection system (Gatan MonoCL4) was used for the IL experiments reported here. The MonoCL4 system consists of a retractable mirror and light guide which can be positioned over the sample to collect the light emitted. A hole in the mirror allows the focused helium ion beam through onto the sample. For all experiments, the beam energy was $\sim 30 \mathrm{keV},+/-1 \mathrm{keV}$ depending on the particular arrangement of atoms at the source. Light emitted from the sample as a result of helium ion bombardment (ionoluminescence) is reflected by the parabolic mirror down the light guide and into the spectrometer housing. In panchromatic mode, the light is allowed to pass straight through to a photo-multiplier tube (PMT) detector. An image can then be formed from the luminescence, pixel by pixel, as the helium ion beam is scanned across an area on the sample. To maximize its signal-to-noise ratio, the PMT is thermoelectrically (Peltier) cooled to $-28^{\circ} \mathrm{C}$, with additional water cooling on the hot side of the Peltier element. Spectral filters can be inserted at the PMT entrance port to form images from light over restricted regions of the spectrum, enabling an RGB composite image to be generated. Alternatively, the system can be operated in parallel spectrum acquisition mode, in which the emitted light is directed through slits and onto a diffraction grating. The angularly-distributed spectrum is then projected onto a thermoelectrically cooled CCD array to generate an intensity-vs.-wavelength plot, the range of which can be varied by rotating the grating.

This work surveys the IL emission characteristics of a range of samples known to exhibit $\mathrm{CL}$ in an SEM, the preparation of which will be described in their respective sections. Samples tested include direct band gap semiconductor materials in the form of light-emitting diode (LED) dies, cerium activated yttrium aluminium garnet (YAG:Ce), CdSe/ZnS core-shell quantum dots and rare-earth doped lanthanum phosphate $\left(\mathrm{LaPO}_{4}\right)$ nanocrystals.

The decay in IL with helium ion dose was characterized by exposing samples to the $\mathrm{He}$ ion beam and collecting a spectrum in parallel spectrum acquisition mode every minute for one 
hour. Each spectrum collection involves a 30 second dark spectrum recording (camera shutter closed) and then a 30 second collection of the light from the sample. These exposure tests were performed on each sample that exhibited IL of a sufficient intensity to collect a spectrum. CL from the direct band gap semiconductor sample was also characterized using the same MonoCL4 system installed on a scanning electron microscope (Carl Zeiss EVO LS25 with a LaB 6 source).

\section{Results and Discussion}

\section{Direct bad gap Semiconductors}

As explained previously, direct band gap semiconductors exhibit intense $C L$ as a result of radiative recombination of electron-beam generated electron-hole pairs across the band gap. A striking demonstration of this can be obtained by collecting panchromatic $\mathrm{CL}$ images from LED dies, prepared by dissolving their packaging in chloroform before mounting onto an SEM stub with silver paint. A sample was prepared from a GaN LED (sapphire substrate) in this way and imaged in secondary electron (SE) and $\mathrm{CL}$ mode in the SEM (Figure 1). The blue, $\mathrm{p}$ type doped GaN active layer is clearly visible and contrasts well with the non-luminescent metal contacts. The yellow emission at the edge is thought to be caused by nitrogen vacancies in the thick n-type GaN layer (Monteiro et al., 1997).
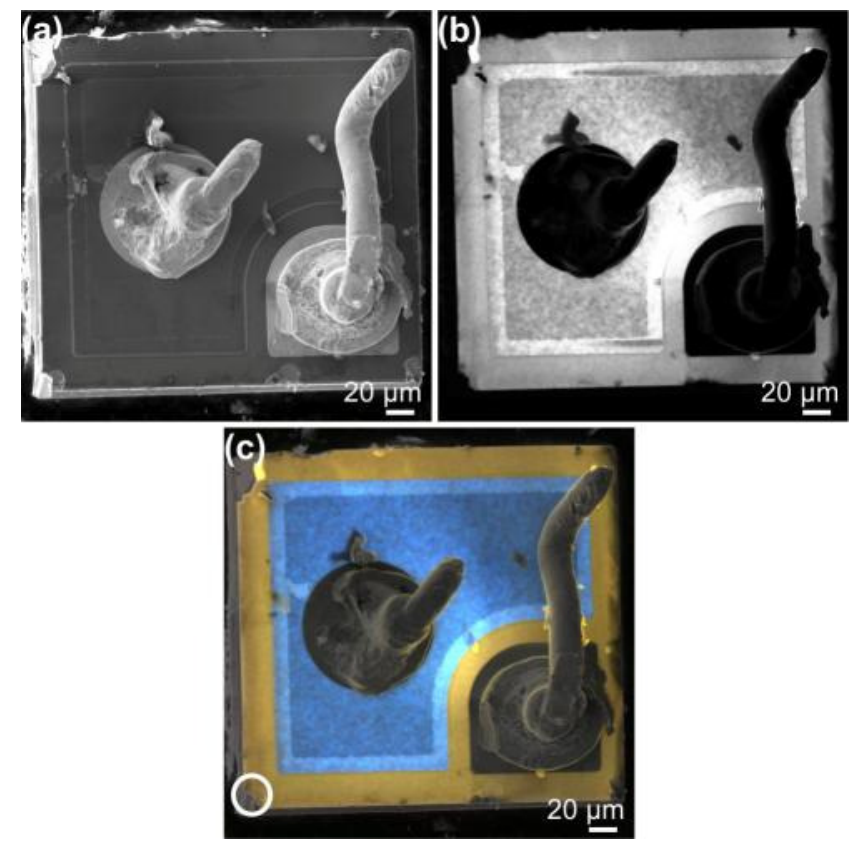

Figure 1. Secondary electron (SE) and CL images of a GaN LED with sapphire substrate in SEM: (a) SE image, (b) panchromatic CL image, (c) RGB-SE composite image.
The GaN LED die was also imaged in the HIM and attempts were made to form a simultaneous SE and IL images (Figure 2). The GaN layer showed no detectable IL, appearing as a black area on the IL images (Figure 2, (b) and (d)). The only luminescence emanated from the sapphire substrate. Figure 2(a) shows a damaged corner of the device with the sapphire substrate exposed. In order to obtain these images the imaging current required was above $150 \mathrm{pA}$ and the PMT was biased to the maximum of $-1500 \mathrm{~V}$. By comparison, the corresponding $\mathrm{CL}$ images taken in the SEM (Figure 1) are at a comparable beam current ( 200 pA) but with a PMT tube bias of only $-597 \mathrm{~V}$. This represents a large amount of $\mathrm{CL}$ compared to IL signal since the effect of doubling the PMT voltage normally increases the detected luminescence by several orders of magnitude. Therefore, even though some luminescence of the sapphire was detected under the ion beam, it is many orders of magnitude lower than that under the electron beam from the $\mathrm{GaN}$ in this sample.
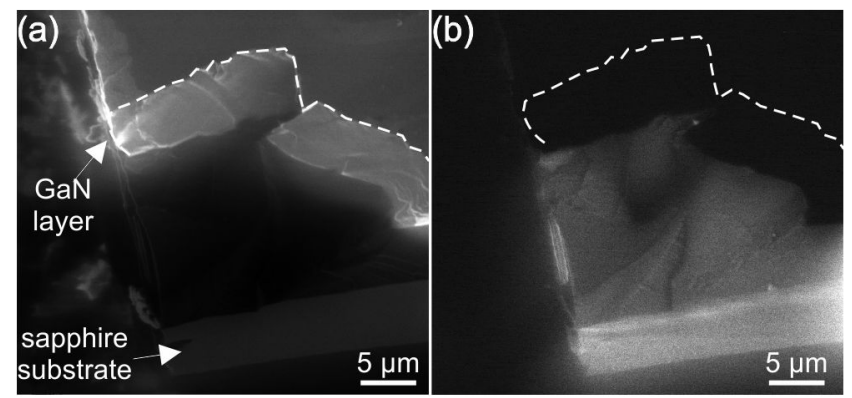

(c)
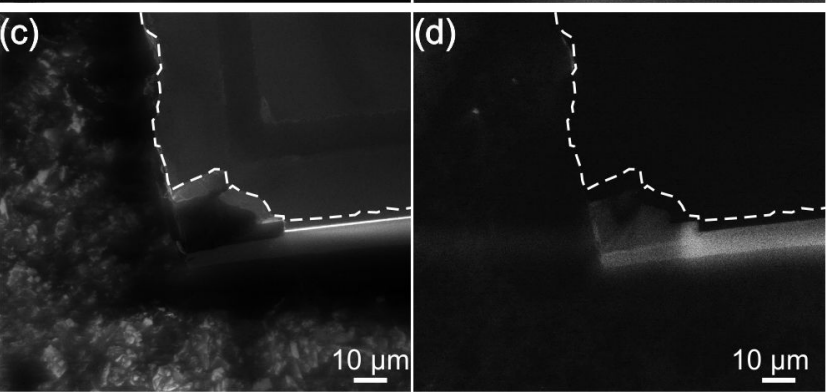

Figure 2. Secondary electron (SE) and IL images of GaN LED with sapphire substrate in HIM: (a) SE image of corner of device shown by white circle in Figure 1(c), (b) corresponding IL image, (c) Low magnification SE image from same corner, (d) IL image corresponding to (c). The white dashed lines mark the top edge of the GaN layer as a guide to the eye.

Luminescence of sapphire, excited via electrons (CL) (Miyauchi \& Shibata, 1993) or He ions (IL) (Jardin et al., 1996), has been studied previously. Jardin et al. have reported strong IL emission of a sapphire sample when irradiated with a $2 \mathrm{MeV}$ $\mathrm{He}^{+}$beam (Jardin et al., 1996). The IL intensity 
initially increases with increasing ion dose due to creation of defects that act as $\mathrm{F}^{+}$centres. As the ion dose increases above $2 \times 10^{16}$ ions $/ \mathrm{cm}^{2}$, IL intensity begins to decrease due to annihilation of luminescence centres by extended defect formation. A conversion of $\mathrm{F}$ to $\mathrm{F}^{+}$centres occurs with increasing dose, which manifests as the growth of the $330 \mathrm{~nm}$ peak at the expense of the $415 \mathrm{~nm}$ peak, due to lattice distortions. Unfortunately, the IL intensity observed in the HIM was not sufficient to obtain a spectrum. The luminescence intensity is expected to be much lower in the HIM, with a $\sim 30 \mathrm{keV}$ beam, compared to the $2 \mathrm{MeV}$ beam used by Jardin et al.. Decay in the emission from the sapphire was observed however, as is evident Figure 2(d), in which the corner of the sample, which was exposed to a higher ion dose for the image in Figure 2(b), is noticeably dimmer than the sides. This is probably due to annihilation of luminescence centres by extended defect formation.

Several other direct semiconductor materials were investigated but no IL was detected. This was unexpected because the secondary electron yield in HIM is high. The formation of images from the SE emission from these materials in the HIM proves that there is sufficient energy to cause electron-hole pair generation which should lead to luminescence by radiative recombination across the direct band gap. The absence of any IL above the detection limits of the system indicates that non-radiative decay processes may be dominant, possibly caused by extended dislocations in the crystal lattice formed by the bombardment of the sample by energetic $\mathrm{He}$ ions. It is well known that ion-implantation can quench luminescence in semiconductors through the formation of non-radiative recombination centres. For example, Kucheyev et al. report that low dose implantation of GaN with light ions in the keV range dramatically quenches visible $\mathrm{CL}$ emission (Kucheyev et al., 2001). This is demonstrated with implantation of $150 \mathrm{keV}{ }^{12} \mathrm{C}^{-}$ ions to a dose of $10^{14} \mathrm{~cm}^{-2}$, a dose that can be achieved in the HIM with a beam current of 100 $\mathrm{pA}$ and a FOV of $100 \mu \mathrm{m}$ in 20 seconds. Although SRIM (Biersack \& Haggmark, 1980; Zeigler et al., 1985) calculations show that $150 \mathrm{keV}$ carbon ions produce approximately 8 times as many vacancies per ion as $30 \mathrm{keV}$ He ions, it is important to note that with luminescence in the $H I M$, the same ions that generate e-h pairs are also generating defects. Therefore, unlike $\mathrm{CL}$ experiments on ion-implanted samples where the electron beam is used to probe the material after the damage has been done, defects in HIM studies will be closer to the generated e-h pairs and, resulting in enhanced non-radiative, defectmediated recombination and so more effective quenching of luminescence.

The volume of material from which luminescence is generated could also be an important factor as to why IL in bulk semiconductors is negligibly small compared to $\mathrm{CL}$. Electron and helium ion trajectory plots were generated using the Monte Carlo simulation packages CASINO (Drouin et al., 2007) and SRIM (Zeigler et al., 1985; Biersack \& Haggmark, 1980), respectively, to examine the different interaction volumes associated with $\mathrm{CL}$ and IL generation (Figure 3). A visual comparison of the two plots in Figure 3 reveals that the interaction volume of a $20 \mathrm{keV}$ electron beam in GaN is roughly 200-250 times that of the $30 \mathrm{keV}$ helium ion beam. This simple comparison only considers the distribution of injected charged particles in the sample, it does not take into account the differences in the generation of luminescence by the two beams or effects of reabsorption of generated photons. Nevertheless, it demonstrates that the electron beam interacts with a volume several orders of magnitude larger than that of the helium ion beam, a factor which could contribute to the larger CL signal compared to IL signal detected from bulk samples. Furthermore, it is well known that $\mathrm{CL}$ emission from near the surface of bulk samples can be reduced by the increased concentration of nonradiative recombination centres and the presence of residual surface electric fields caused by Fermi level pinning, creating an optically inactive so called "dead layer" (Abrams \& Holloway, 2004). Indeed, this has been shown to lead to a decrease in $\mathrm{CL}$ intensity with beam energy: $\mathrm{A}$ larger proportion of the interaction volume for a low energy electron beam is within the surface dead layer and therefore the $\mathrm{CL}$ intensity is lower compared to a high energy electron beam (Seager et al., 1997). The Monte Carlo simulation results show that the interaction volume for the $\mathrm{He}$ ion beam is closer to the surface and so a larger proportion will be within the dead layer, 
where luminescence is effectively quenched, compared to the electron beam which interacts with material deeper in the sample
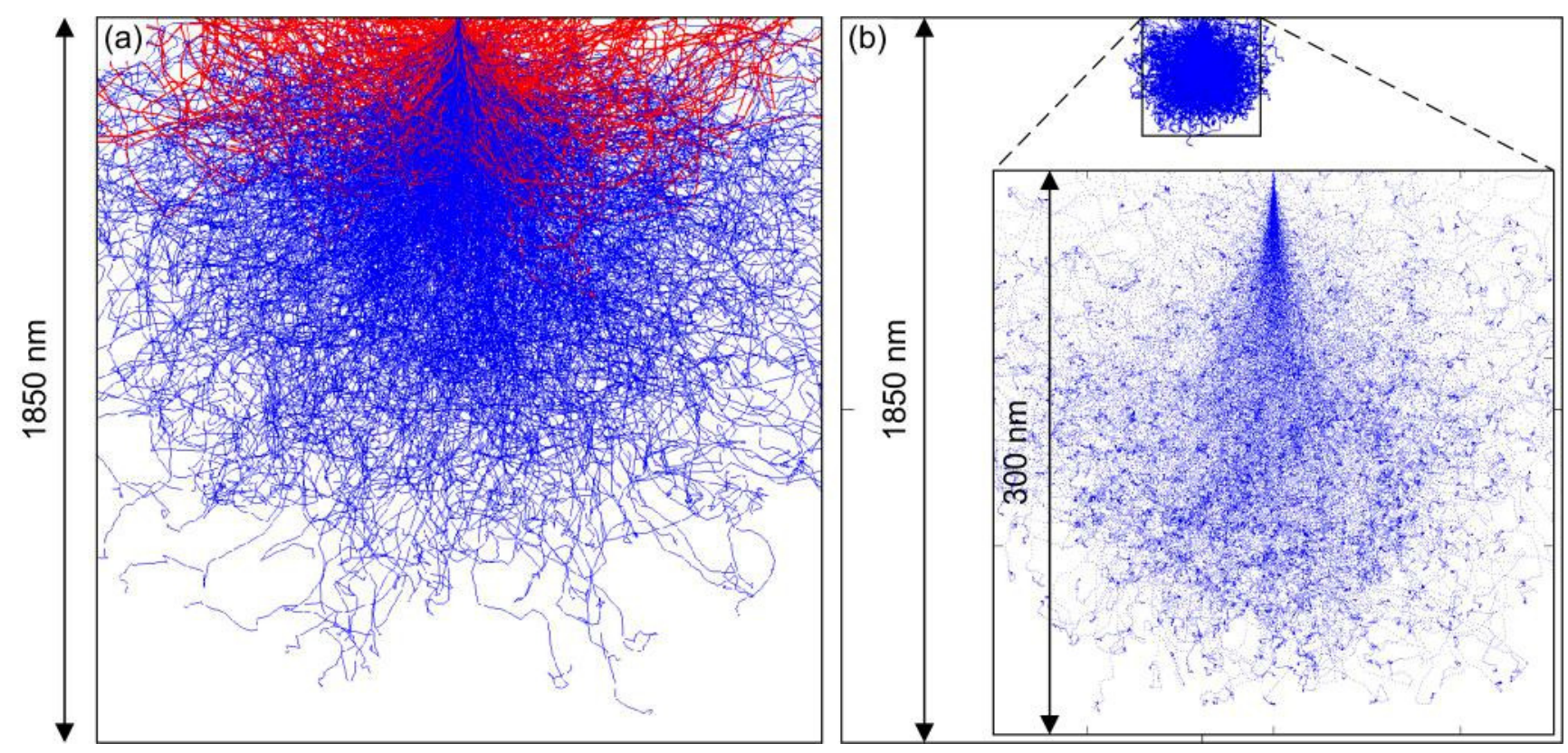

Figure 3. Monte Carlo simulation results showing charged particle trajectories through GaN: (a) 20 keV electrons simulated using CASINO (red tracks are backscattered electrons, blue tracks are electrons remaining in the sample); (b) $30 \mathrm{keV}$ helium ions simulated using SRIM.

\section{Rare-earth doped garnet}

Yttrium aluminium garnet activated with cerium (YAG:Ce) is a phosphor that finds applications in white LEDs because of its ability to efficiently convert blue LED emission to broadband yellow light (Pan et al., 2004). It is also known to exhibit intense $\mathrm{CL}$ as a result of $5 \mathrm{~d}-4 \mathrm{f}$ transitions in the $\mathrm{Ce}^{3+}$ ions (Yuan et al., 1989; Jang et al., 2007).

A polished disk of YAG:Ce approximately $8 \mathrm{~mm}$ in diameter and $0.5 \mathrm{~mm}$ thick was mounted onto an Al SEM stub using silver paint. Panchromatic images and spectra were collected and an exposure test to assess the luminescence decay over time was performed at a low magnification. Low magnification images of the top surface of the YAG:Ce sample, taken at the edge of the disk, are shown in Figure 4 (a-c). The garnet is insulating and so charging effects lead to a low SE signal in Figure 4a in which the stub surface at the top of the image appears bright and the YAG:Ce in the lower part of the image appears dark. The HIM is supplied with an electron flood gun that can be used to neutralize charge build-up but this process is incompatible with collection of IL because the filament glow from the flood-gun would mask any IL from the sample. The IL emission spectrum for this sample (Figure 4 (d)) shows broadband emission centred on a wavelength of $555 \mathrm{~nm}$. This agrees well with $\mathrm{CL}$ spectra of similar samples from the literature (Yuan et al., 1989; Jang et al., 2007). The IL signal from the YAG:Ce sample is sufficiently large that at low magnifications, a bright spot is observed in the IL image (Figure 4(b)) corresponding to the area on the sample from which the mirror most efficiently collects the light. For efficient operation, the mirror has to be adjusted so that this spot is in the centre of the FOV and so the YAG:Ce sample is useful for alignment of the mirror before attempting to collect IL data from other samples.

An exposure test was performed on this sample, with a FOV of $450 \mu \mathrm{m}$, a beam current of approximately $0.6 \mathrm{pA}$ and so a dose rate of $1.85 \times 10^{9}$ ions $/ \mathrm{cm}^{2} / \mathrm{s}$. The data were smoothed using a moving average smoothing function (Figure 5 (a)); the peak in emission at $553 \mathrm{~nm}$ has been extracted and plotted in Figure 5(b). After an initial drop in luminescence up to a dose of $10^{13}$ ions $/ \mathrm{cm}^{2}$, the IL intensity appears to level out 
and even rise slightly before decreasing again as the dose reaches $\sim 1.7 \times 10^{13}$ ions $/ \mathrm{cm}^{2}$. A continuous decrease is expected with increasing dose as luminescent centres are quenched and so this anomalous rise could be due to instabilities caused by sample charging. The decay in luminescence with exposure is also observed in the images: The dark patches at the edge of the sample in the right hand side of the IL images in Figure 4(b) and (c) (marked by the red circles in Figure $4(\mathrm{~b}))$ are areas previously exposed to higher doses of helium ions (i.e. imaged at higher magnifications) before the present images were obtained.
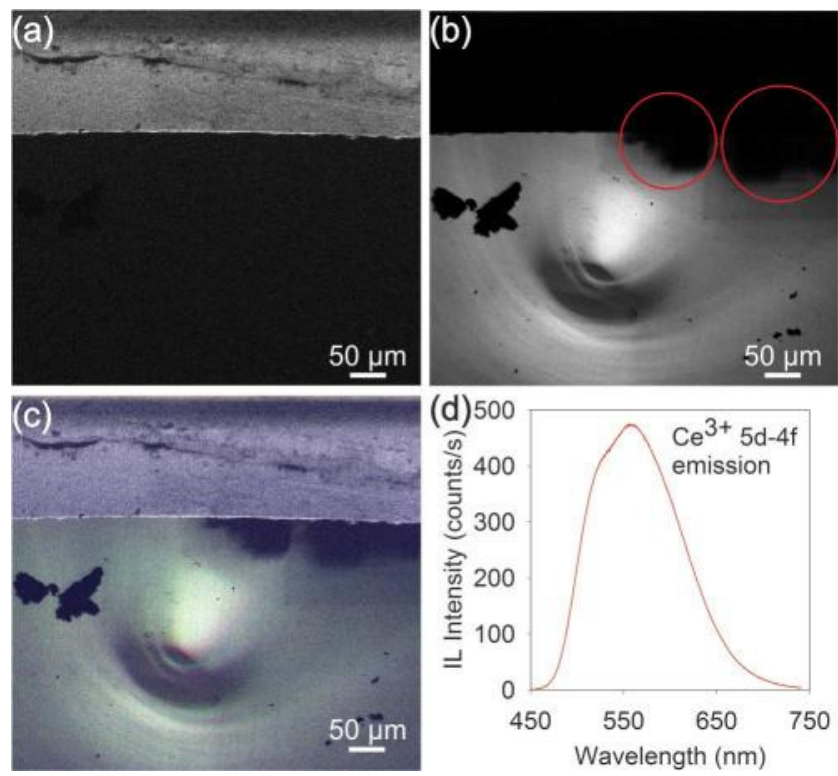

Figure 4. HIM Images and IL spectrum from edge of YAG:Ce sample: (a) SE image; (b) IL image; (c) combined RGB IL and SE image; (c) IL spectrum. Previously exposed areas are marked by red circles in (b).

Samples for fluorescent bio-tagging applications

A key potential application for IL in the helium ion microscope is the acquisition of simultaneous SE and IL images to map the location of the fluorescently-labelled molecules on a biological cell (Fisher et al., 2010; Fisher et al., 2008). Cathodoluminescence in an SEM has been used in this way, in which antibodies conjugated to particles or molecules which fluoresce under the electron beam (fluorophores) are allowed to bind to specific sites on a cell. Electron beam induced emission from the fluorophores is then used to identify the location of these sites with respect to the rest of the cell. Recently, inorganic fluorophores based on semiconductor quantum dots and rare earth doped nanoparticles have been developed that are more stable and exhibit a higher quantum yield under an electron beam than traditional organic dye fluorophores (Fisher et al., 2010). To assess their suitability as fluorophores for IL imaging, images and spectra were acquired from examples of these materials.
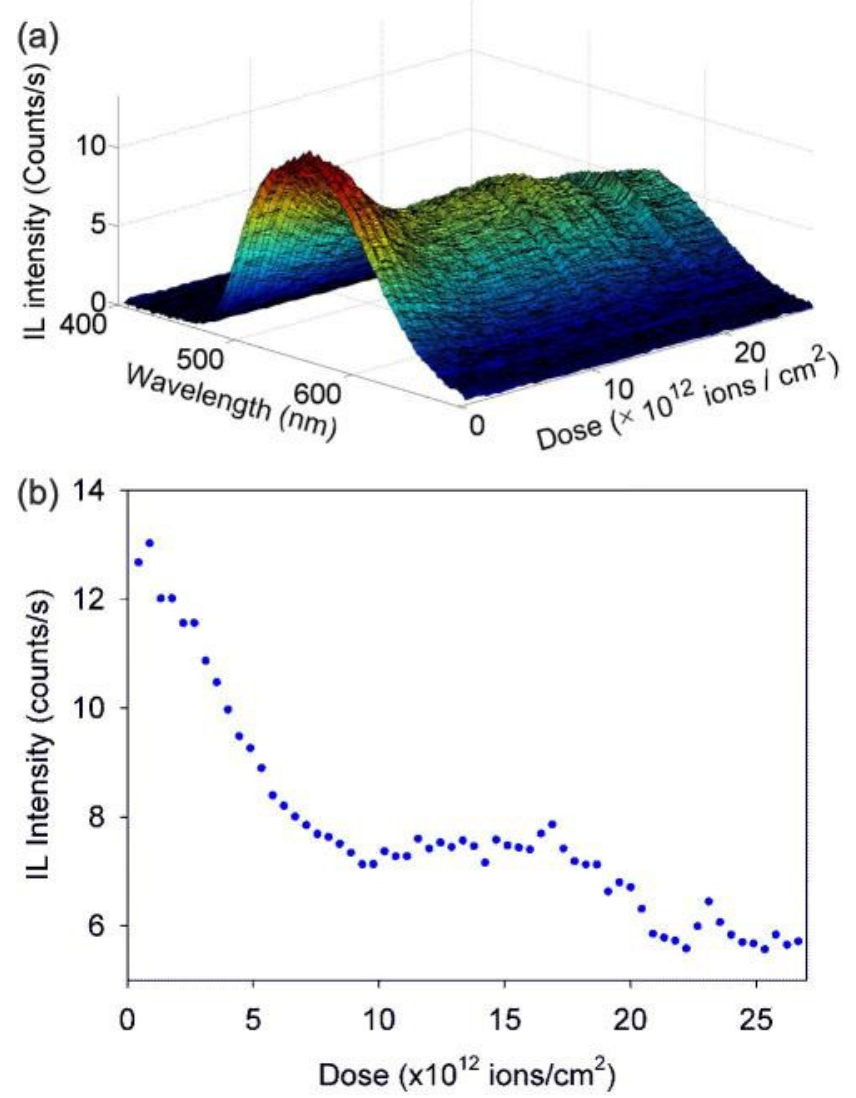

Figure 5. Graphs showing IL decay with exposure to the beam for the YAG:Ce sample. (b) shows a cross section through (a) at the $553 \mathrm{~nm}$ peak.

\section{CdSe/ZnS Quantum Dots}

A drop of CdSe/ZnS core shell quantum dots in solution (Lumidot ${ }^{\mathrm{TM}} 610$, Sigma Aldrich), with an emission peak wavelength of $610 \mathrm{~nm}$, was deposited onto an aluminium SEM stub and left to dry overnight. Simultaneous SE and panchromatic IL images were obtained in the HIM at low magnifications (Figure 6(a) and (b)), clearly showing luminescence from the agglomerated material. The RGB composite image (Figure 6(c)) shows this emission is predominantly red and the spectrum confirms the expected characteristic red emission with a peak at $623 \mathrm{~nm}$. This is shifted compared to the reported emission of the quantum dots in solution $(610 \mathrm{~nm})$, probably due to surface interactions and agglomeration of the particles.

Attempts to image the quantum dot agglomerates at higher magnifications were unsuccessful due to the rapid quenching of 
luminescence with ion beam exposure. An exposure test was performed and the luminescence is seen to decay to below 1 count per second despite the low dose rate $\left(\sim 7.3 \times 10^{10}\right.$ ions $/ \mathrm{cm}^{2} / \mathrm{s}$ ), as shown in Figure 7. The decay results fit well to a two phase exponential function, of the form $y=a e^{b x}+c e^{d x}$, suggesting that two different mechanisms are contributing to the luminescent decay, although the nature of these mechanisms is currently unclear.
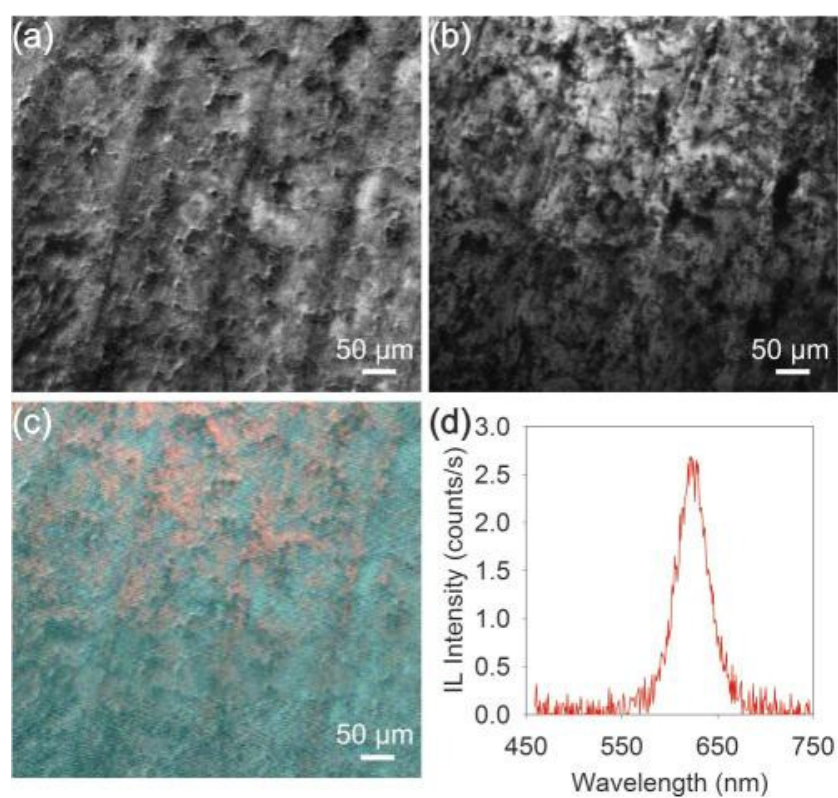

Figure 6. HIM Images and IL spectrum from the CdSe/ZnS QD sample: (a) SE image; (b) IL image; (c) combined RGB IL and SE image; (d) IL spectrum. The PMT bias was -1158 V and the beam current was $42.1 \mathrm{pA}$.

The detection of IL with the QD sample when no IL could be detected from the bulk semiconductor samples could be due to the small size of the individual $Q D$ particle. For $I L$ in a $Q D$ to be quenched, the incident beam would need to displace atoms within the QD, forming defects which would act as non-radiative recombination centres. Studies have shown that the ion beam generated in the HIM interacts primarily with the sample electrons at first, with collision cascades through nuclear interactions occurring deeper in the material (Cohen-Tanugi \& Yao, 2008). Therefore it is likely that defects within QDs would be generated at a lower rate than defects within bulk semiconductors, rendering the QD sample more resistant to quenching as observed here. (a)
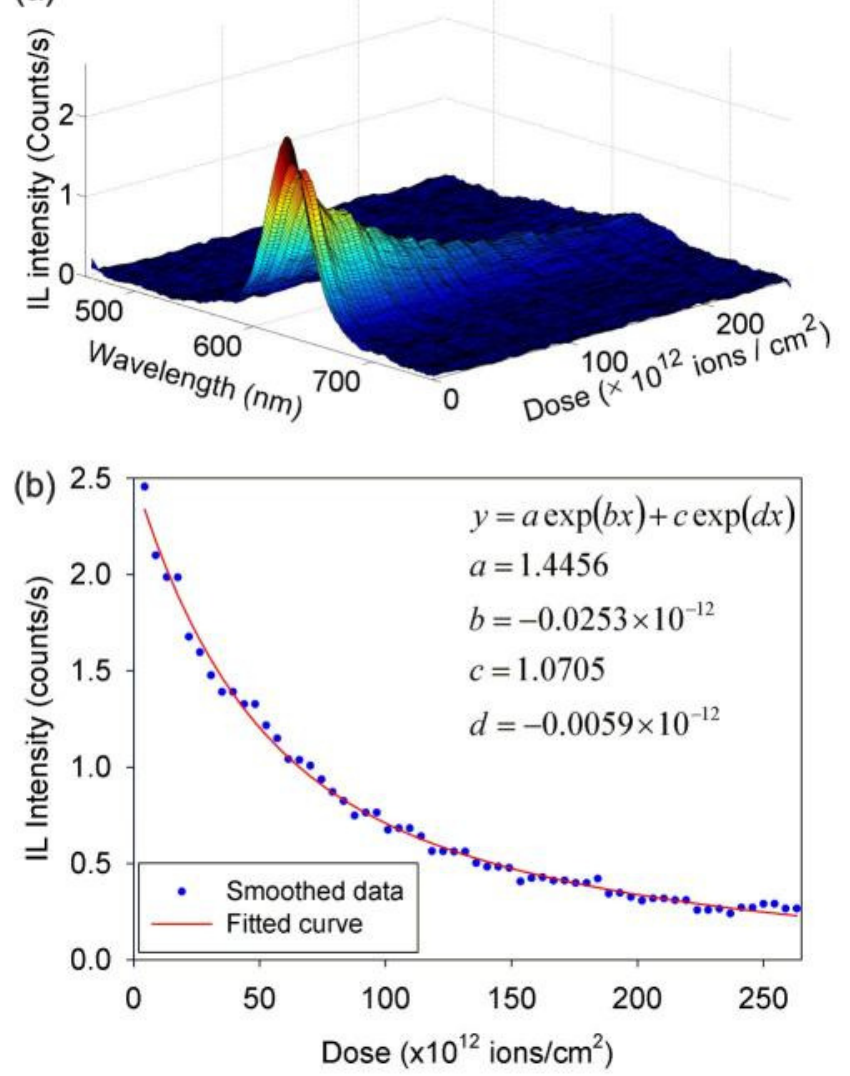

Figure 7. Graphs showing IL decay with exposure to the beam for the CdSe/ZnS QD sample. (b) shows a cross section through (a) at the $621 \mathrm{~nm}$ peak, fitted to a double exponential function.

To shed further light on this, Monte Carlo simulations were performed using SRIM. The diameter of the dots quoted by the manufacturers is $5.2 \mathrm{~nm}$ and so they were modelled in SRIM as a $5.2 \mathrm{~nm}$ thick layer of CdSe on an Al substrate. The interaction of 50,000 30 $\mathrm{keV}$ ions was simulated and the resulting histograms of the total vacancy generation and $\mathrm{He}$ ion distribution are shown in Figure 8. The simulations show that the only $0.8 \%$ of the total vacancies are generated in the CdSe layer and only $0.04 \%$ of the He ions come to rest in the CdSe layer. The vast majority of the damage occurs in the aluminium substrate, peaking at a depth of around $200 \mathrm{~nm}$. This represents only the ideal case of a monolayer of QDs on a substrate and does not account for the aggregation of QDs. Nevertheless it serves as an illustration of the relatively small proportion of $\mathrm{He}$ ion generated defects in quantum dots compared to those that would be generated in bulk semiconductors and so offers an explanation for the lower quenching rate observed. 


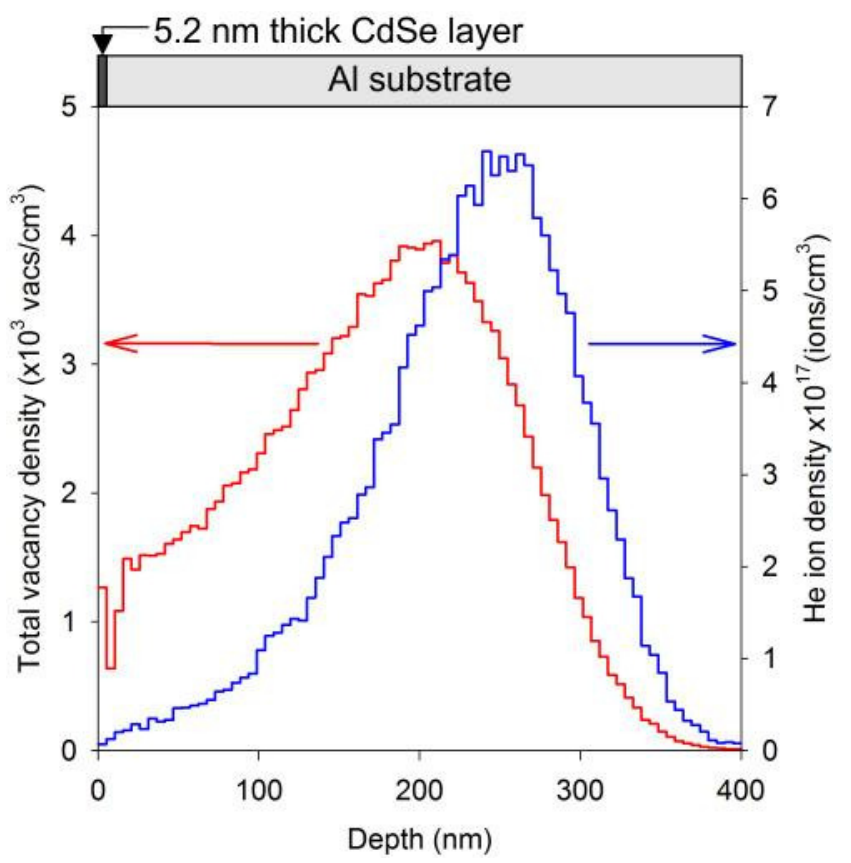

Figure 8. Histograms of total vacancy density (from He ion knock-ons and from collision cascades) and He ion density following the SRIM simulation of 50,000 ions interacting with a $5.2 \mathrm{~nm}$ thick layer of CdSe on an Al substrate. The units were calculated for an ion dose of $10^{13}$ ions $/ \mathrm{cm}^{2}$.

\section{Rare earth doped lanthanum phosphate nanoparticles}

Rare Earth doped lanthanum phosphate $\left(\mathrm{LaPO}_{4}\right)$ nanocrystals (K. Riwotzki et al., 2000) are a class of luminescent nanoparticle with similar dimensions to QDs but with a different mechanism responsible for their luminescence. QD luminescence originates from transitions across size dependent band gaps whereas the luminescence in doped $\mathrm{LaPO}_{4}$ nanocrystals is a result of transitions between $f$ electron states. These are bulk properties and so are independent of the size of the nanocrystals. They are known to exhibit quantum yields up to $61 \%$ (Riwotzki et al., 2001), are highly stable and have a low toxicity, making them promising candidates for biotagging applications (Schuetz \& Caruso, 2002).

Two samples of rare earth doped $\mathrm{LaPO}_{4}$ nanocrystals dispersed in an aqueous solution (REN-XR/REN-XG, Nanogate, Inc.) were investigated:

- Eu doped $\mathrm{LaPO}_{4}$ (REN-XR, red emitters).

- $\mathrm{Ce} / \mathrm{Tb}$ doped $\mathrm{LaPO}_{4}$ (REN-XG, green emitters).

Drops of the two solutions were deposited on aluminium stubs and allowed to dry. The samples were imaged in SE and IL modes at low magnification and RGB composite images were generated (Figure 9 and Figure 10, (a-c)). These images clearly show luminescence from clumps of agglomerated particles, with the RGB composite images revealing red emission from $\mathrm{Eu}$ doped particles and green emission from $\mathrm{Ce} / \mathrm{Tb}$ doped particles. Spectra of the emission from the doped $\mathrm{LaPO}_{4}$ samples reveal emission peaks corresponding to f-electron transitions in the rare-earth dopants (Figure 9 and Figure 10, (d)). The Eu doped $\mathrm{LaPO}_{4}$ nanocrystals emit light because $f$ electrons in the $\mathrm{Eu}^{3+}$ dopants gain energy and are excited into the $D$ states. Here they undergo non-radiative energy loss to the ${ }^{5} D_{0}$ state and then emit a photon and drop down to an $\mathrm{F}$ state, producing the spectral peaks shown in Figure $9(d)$, the positions of which correspond to photoluminescence (PL) results from Riwotzki et al. (Riwotzki et al., 2000). The same applies to emission from $\mathrm{Ce} / \mathrm{Tb}$ doped $\mathrm{LaPO}_{4}$ particles, where the peaks correspond to the $f$ electron transitions in $\mathrm{Tb}^{3+}$ from the state labelled ${ }^{5} \mathrm{D}_{4}$ in the PL spectrum from Riwotzki et al. (Riwotzki et al., 2001).

As with the other samples studied, the luminescence from the rare-earth doped nanoparticle samples is observed to decay during exposure to the helium beam. Results from exposure tests on the two samples are presented in Figure 11. The decay curves in Figure 11 fit well to double exponential functions, suggesting two components to the quenching of luminescence.

As with CL (Maqbool et al., 2012), it is thought that there are two mechanisms of excitation for IL in rare-earth doped material: Direct and indirect. For direct excitation, energy is transferred from an incident helium ion to an $f$ electron in a rare-earth impurity, exciting the electron into a $D$ state where it undergoes nonradiative energy loss to the lowest $D$ state and then radiatively decay back to an $\mathrm{F}$ state. Indirect excitation occurs via the generation of mobile electron-hole pairs (EHPs) in the host crystal, which diffuse and can recombine radiatively at the rare-earth centres. One explanation of the observed decay in IL with time is that the indirect excitation of RE by EHPs will be quenched by defect formation due to collisions of the He ions with the crystal atoms. These defects will be centres for non-radiative recombination and so as there number increases over time, the number of 
EHPs recombining radiatively at $\mathrm{RE}$ centres will reduce, leading to a reducing in IL intensity.
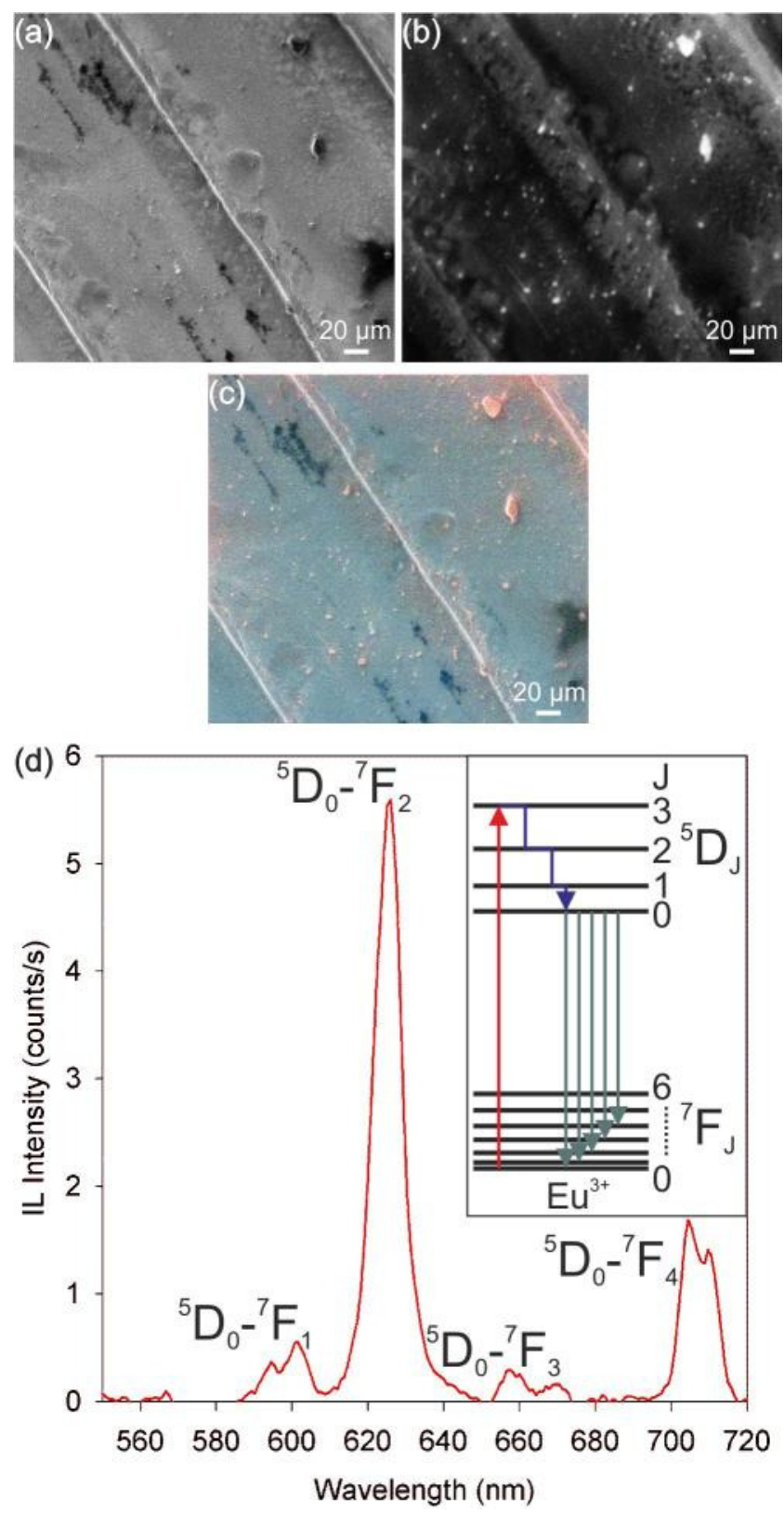

Figure 9. HIM Images and IL spectrum from edge of REN-

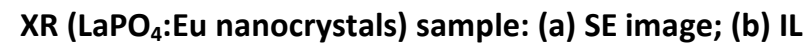
image; (c) combined RGB IL and SE image. The PMT bias was $-1047 \mathrm{~V}$ and the beam current was $38.9 \mathrm{pA}$. (d) IL spectrum with inset illustrating f electron transitions in the $\mathrm{Eu}^{3+}$ dopant ions.
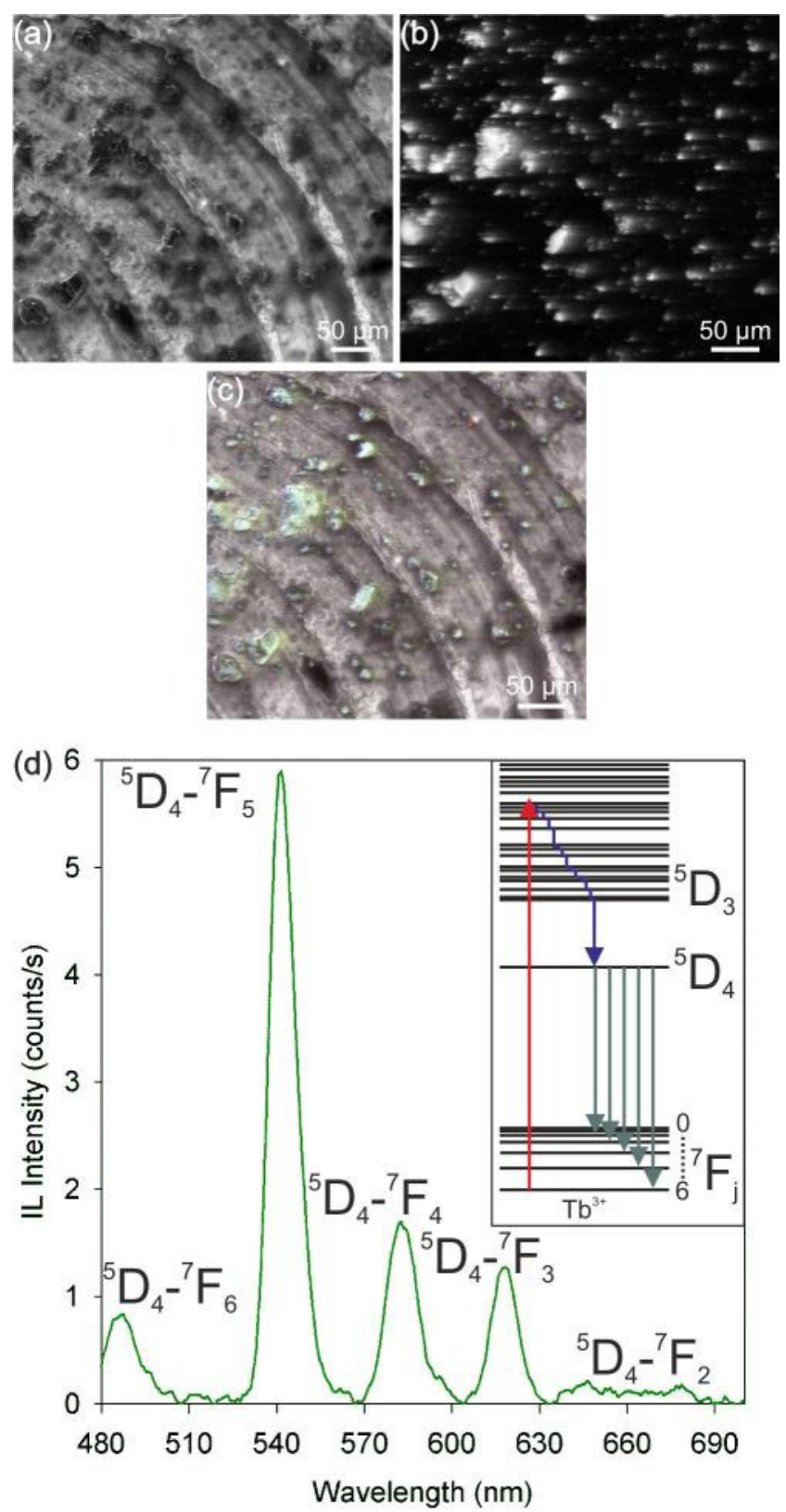

Figure 10. HIM Images and IL spectrum from edge of RENXG (LaPO ${ }_{4}: \mathrm{Ce} / \mathrm{Tb}$ nanocrystals) sample: (a) SE image; (b) IL image; (c) combined RGB IL and SE image. The PMT bias was $-1024 \mathrm{~V}$ and the beam current was $33.4 \mathrm{pA}$. (d) IL spectrum with inset illustrating $f$ electron transitions in the $\mathrm{Tb}^{3+}$ dopant ions. 

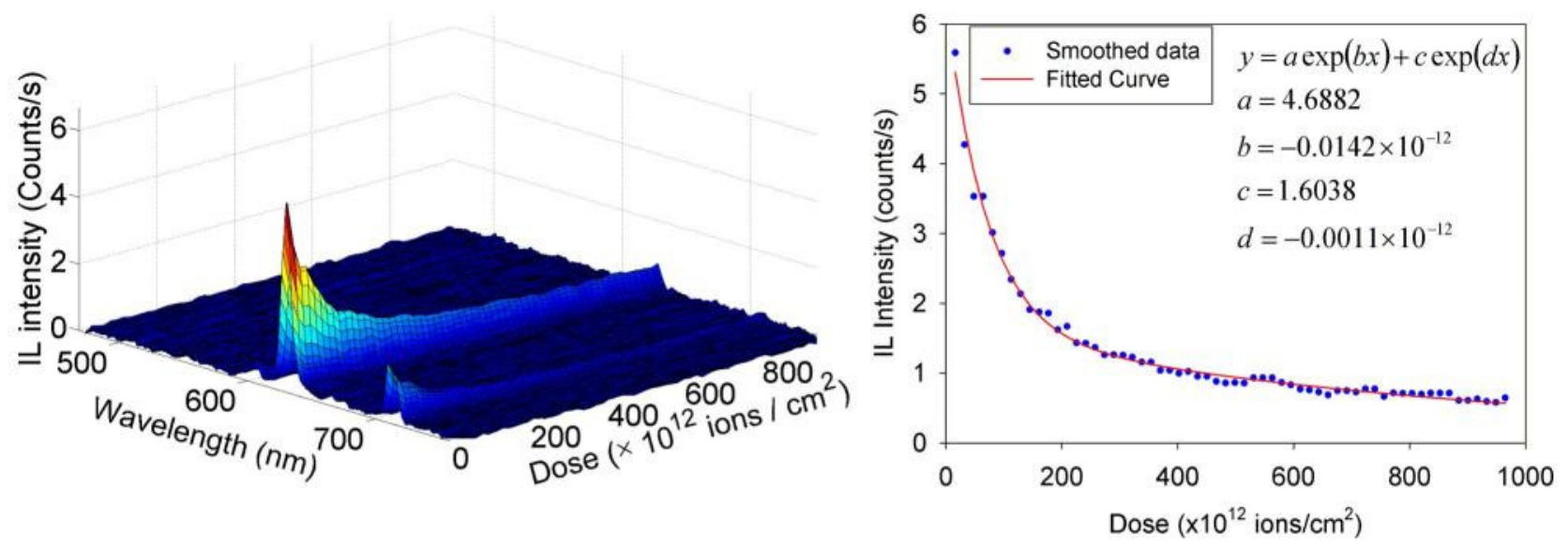

(c)

(d)
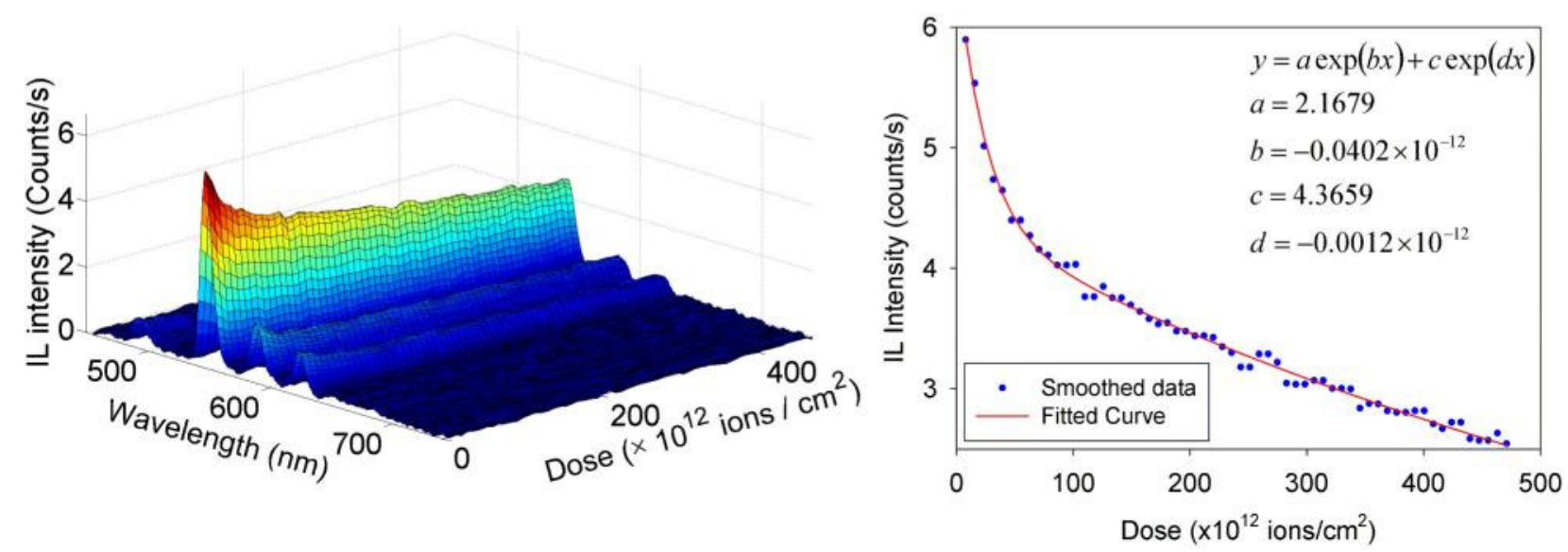

Figure 11. Graphs showing IL decay with exposure to the beam for the LaPO4 nanocrystal samples doped with (a), (b) Eu and (c), ( d) $\mathrm{Ce} / \mathrm{Tb}$. (a \& c) Surface plots showing the change in emission spectra as the sample is exposed to the helium beam. (b \&

d) Cross sections through surface plots in (a) and (c) at the (b) $542 \mathrm{~nm}$ peak, and (d) $626 \mathrm{~nm}$ peak. Double exponential functions are fitted to each peak decay curve, with the parameters of the fits also shown. Beam currents and fields of view: (a

\& b) $38.6 \mathrm{pA}$ and $300 \mu \mathrm{m}$, giving a dose rate of $2.86 \times 10^{11} \mathrm{ions} / \mathrm{cm}^{2} / \mathrm{s}$. (c \& d) $33.5 \mathrm{pA}$ and $400 \mu \mathrm{m}$, giving a dose rate of $1.31 \times 10^{11}$ ions $/ \mathrm{cm}^{2} / \mathrm{s}$.

In $\mathrm{CL}$ studies on phosphors, it has been shown that charge build up at the sample surface during exposure to the electron beam can cause a decrease in luminescent intensity (Seager et al., 1997). This Coulomb aging effect is explained as being due to a decrease in the arrival energy of incident electrons as they decelerate in the field caused by the negative surface charge. Likewise, during helium ion bombardment, surfaces can acquire a positive charge due to positive ions entering and secondary electrons exiting the material. However, as the mass of a helium ion is over 7000 times that of an electron, the relative effect of surface charging on arrival energy will be much smaller. Other possible mechanisms identified for the observed Coulomb aging in phosphors include increases in the density of non-radiative pathways at the surface and changes in local electric fields affecting the motion of electrons and holes (Seager et al., 1997). For IL, these could be important quenching mechanisms as the lattice damage to the surface, as well as in the rest of the interaction volume, is higher under ion compared to electron bombardment.

Note that direct comparisons of the intensity of the emission from the two types of $\mathrm{LaPO}_{4}$ particles and the quantum dot samples cannot be made because the amount of material within a field of view varies between samples and exposures.

Higher magnification SE and IL imaging on agglomerations of the rare-earth doped nanocrystals was attempted (Figure 12). A $20 \mu \mathrm{m}$ aperture was used to reduce the beam current to $\sim 1$ pA (to limit IL quenching) and to reduce the probe size and so increase the resolution of the 
images. The images in Figure 12 demonstrate that IL imaging on the sub-cellular scale is possible with this technique, a promising result for biotagging applications. However, these images were obtained from thick agglomerations of particles whereas if these nanocrystals were used to label cells, the density of luminescent centres would be much lower, leading to a substantially lower IL signal. In addition, the SE images suffer from a low signal-to-noise ratio because the large collecting mirror absorbs many of the SEs emitted from the sample, reducing the number that reaches the ET detector. Therefore, better results may be possible by first collecting the IL image of an area and then removing the mirror and collecting the SE image, rather than attempting to collect the two images simultaneously.
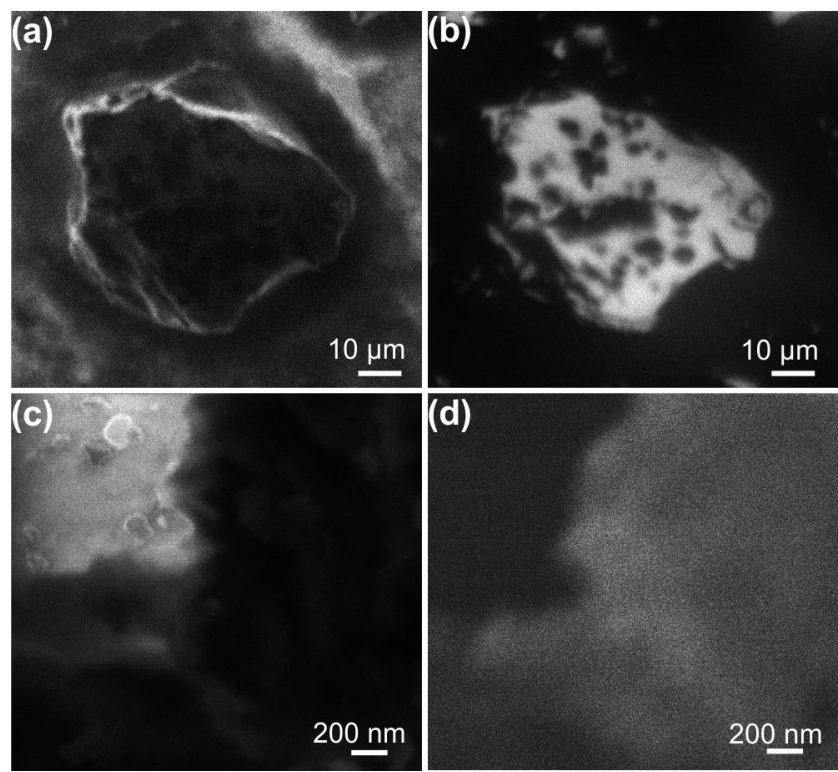

Figure 12. High magnification imaging of $\mathrm{LaPO}_{4}: \mathrm{Ce} / \mathrm{Tb}$ nanocrystal agglomerates (a \& c) HIM SE images; (b \& d) simultaneously acquired IL images. The PMT bias was $1012 \mathrm{~V}$ and the beam current was $0.9 \mathrm{pA}$.

\section{Conclusion}

A helium ion microscope combined with a luminescence detection system has been used to study ionoluminescence in a number of materials systems. Direct band gap semiconductors that show strong cathodoluminescence in an SEM were found not to exhibit detectable ionoluminescence in a HIM. This is possibly due to a combination of the small interaction volume, a large proportion of which could sit in the optically inactive dead layer near the surface, and the impinging ion beam creating defects in the material which provide non-radiative recombination centres near to the excited electrons, causing effective quenching of any luminescence. Quantum dots show weak ionoluminescence corresponding to the expected band gap transition, which decays with exposure to the beam. Quantum dots may be more resistant to quenching than bulk semiconductors as their small size reduces the likelihood of luminescence-quenching beam-induced defects forming within the dots. Rare-earth doped samples are also found to exhibit IL, both in bulk form, as demonstrated with a cerium doped YAG sample, and in nanoparticle form in $\mathrm{Eu}$ and $\mathrm{Ce} / \mathrm{Tb}$ doped $\mathrm{LaPO}_{4}$ specimens.

Emission spectra from these samples reveal emission peaks corresponding to $f$ electron transitions within the rare-earth dopants. Decay of luminescence with beam exposure is again observed, fitting well to double exponential functions which indicates the operation of two decay mechanisms, the details of which are not currently understood. The rare earth doped $\mathrm{LaPO}_{4}$ material shows promise for bio-tagging applications, with IL images acquired at the subcellular scale. However, the IL intensity may not be sufficient when these particles are used to label cells as the particle density is likely to be much lower than in the agglomerates studied here. Future work should focus on further exposure tests at different dose rates to elucidate the nature of the luminescent decay mechanisms at work with these samples and a comparison with $\mathrm{CL}$ emission from the same samples. Methods of dispersing the particles onto a substrate are required for characterization of emission from individual particles, to assess the viability of the technique for bio-tagging applications.

\section{Acknowledgements}

The authors would like to thank Dr David Stowe from Gatan, Inc. for useful discussions.

\section{References}

Abrams, B. L. \& Holloway, P. H. (2004). Role of the surface in luminescent processes. Chemical reviews 104, 5783-801. 
BieRSACK, J. P. \& HAgGMARK, L. G. (1980). A Monte Carlo computer program for the transport of energetic ions in amorphous targets. Nuclear Instruments and Methods in Physics Research 174, 257-269.

Cohen-TAnugl, D. \& YaO, N. (2008). Superior imaging resolution in scanning helium-ion microscopy: A look at beam-sample interactions. Journal of Applied Physics 104, 063504.

Drouin, D., Couture, A. R., Joly, D., TAStet, X., AimeZ, V. \& GAUVIN, R. (2007). CASINO V2.42: a fast and easy-to-use modeling tool for scanning electron microscopy and microanalysis users. Scanning 29, 92-101.

Fisher, P. J., WeSSELS, W. S., Dietz, A. B. \& PRendergast, F. G. (2010). Immunofluorescence scanning electron microscopy of biological cells. Microscopy Today 18, 813.

Fisher, P.J., Wessels, W.S, Dietz, A. B. \& PRENDERGASt, F.G. (2008). Enhanced biological cathodoluminescence. Optics Communications 281, 1901-1908.

Hastenrath, M. \& Kubalek, E. (1982). Timeresolved cathodoluminescence in scanning electron microscopy. In Scanning Electron Microscopy, Johari, O. (Ed.), p. 157. Chicago, IL: SEM, Inc.

JANG, H. S., KANG, J. H., Won, Y.-H., LeE, S. \& JeON, D. $Y$. (2007). Mechanism for strong yellow emission of $\mathrm{Y}_{3} \mathrm{Al}_{5} \mathrm{O}_{12}: \mathrm{Ce}^{3+}$ phosphor under electron irradiation for the application to field emission backlight units. Applied Physics Letters 90, 071908.

JARDIN, C., CANUT, B. \& RAMOS, S. (1996). The luminescence of sapphire subjected to the irradiation of energetic hydrogen and helium ions. Journal of Physics D: Applied Physics 29, 2066-2070.

KuCheyeV, S. O., TOth, M., PhilliPS, M. R., Williams, J. S., JAGADISH, C. \& LI, G. (2001).

Cathodoluminescence depth profiling of ion- implanted GaN. Applied Physics Letters 78, 34.

MAQBOOL, M., JADWISIENCZAK, W. M. \& KORDESCH, M. E. (2012). Cathodoluminescence from amorphous and nanocrystalline nitride thin films doped with rare earth and transition metals. In Cathodoluminescence, Yamamoto, N. (Ed.), pp. 161-205. InTech.

MiYAUCHI, M. \& SHIBATA, N. (1993).

Cathodoluminescence measurement of surfaces in reflection high-energy electron diffraction experiments. Japanese Journal of Applied Physics Part 2 Letters 32, 11791181.

Monteiro, T., Pereira, E., Correia, M., Xavier, C., Hofmann, D., Meyer, B., Fischer, S., Cremades, A. \& PIQUERAS, J. (1997). Broad emission band in $\mathrm{GaN}$ epitaxial layers grown on $6 \mathrm{H}-\mathrm{SiC}$ and sapphire. Journal of Luminescence 72, 696700.

PAN, Y., WU, M. \& SU, Q. (2004). Comparative investigation on synthesis and photoluminescence of YAG:Ce phosphor. Materials Science and Engineering B 106, 251-256.

PenNyCOOK, S. J. (2008). Investigating the optical properties of dislocations by scanning transmission electron microscopy. Scanning 30, 287-98.

Riwotzki, K., MeYsSAMY, H., KoRnOWSKI, A. \& HAASE, M. (2000). Liquid-phase synthesis of doped nanoparticles: Colloids of luminescing $\mathrm{LaPO}_{4}$ :Eu and $\mathrm{CePO}_{4}: \mathrm{Tb}$ particles with a narrow particle size distribution. Journal of Physical Chemistry B 104, 2824-2828.

Riwotzki, K., Meyssamy, H., SCHnablegger, H., KORNOWSKI, A. \& HAASE, M. (2001). Liquidphase synthesis of colloids and redispersible powders of strongly luminescing $\mathrm{LaPO}_{4}: \mathrm{Ce}, \mathrm{Tb}$ nanocrystals. Angewandte Chemie 40, 573-576.

SCHUETZ, P. \& CARUSO, F. (2002). Electrostatically assembled fluorescent thin films of rareearth-doped lanthanum phosphate 
nanoparticles. Chemistry of Materials 14, 4509-4516.

SCIPIONI, L. (2008). Carl Zeiss Application Note: Ultra-High Resolution Imaging by Orion PLUS, available at: http://www.zeiss.de/C1256E4600307C70/E mbedTitellntern/EN_40_011_026_orion_ultr a-

high_resolution.pdf/\$File/EN_40_011_026_ orion_ultra-high_resolution.pdf (accessed 30th June 2012)

Scipioni, L., Stern, L. A., Notte, J., SiJBrandiJ, S. \& GrIFFIN, B. (2008). Helium ion microscope. Advanced Materials \& Processes 166, 27-30.

SCIPIONI, L., Stern, L. \& NotTe, J. A. (2007).

Applications of the helium ion microscope. Microscopy Today 15, pp12-14.

Seager, C. H., Warren, W. L. \& TAllant, D. R. (1997). Electron-beam-induced charging of phosphors for low voltage display applications. Journal of Applied Physics 81, 7994.

SMITH, J. V. \& STENSTROM, R. C. (1965). Electronexcited luminescence as a petrologic tool. Journal of Geology 73, 627-635.

Vanden Berg-Foels, W. S., SCIPIONI, L, Huynh, C. \& WEN, X. (2012). Helium ion microscopy for high-resolution visualization of the articular cartilage collagen network. Journal of microscopy 246, 168-76.

Wakefield, B., Eaves, L., Prior, K., Nelson, A. \& DAVIES, G. (1984). The $1.36 \mathrm{eV}$ radiative transition in InP: its dependence on growth conditions in MBE and MOCVD material. Journal of Physics D: Applied Physics 17, L133-L136.

Ward, B. W., Notte, J. A. \& Economou, N. P. (2006). Helium ion microscope: A new tool for nanoscale microscopy and metrology. Journal of Vacuum Science and Technology $B$ 24, 2871-2874.

YACOBI, B. \& HOLT, D. (1986). Cathodoluminescence scanning electron microscopy of semiconductors. Journal of Applied Physics 59, R1-R24.

YUAN, J., BERGER, S. \& BROWN, L. M. (1989).

Thickness dependence of cathodoluminescence in thin films. Journal of Condensed Matter 1, 3253-3265.

ZEIGLER, J. F., BIERSACK, J. P. \& LITTMARK, U. (1985). The stopping and range of ions in solids. New York: Pergamon Press. 